Conclusions Approaches adopted to distribute the survey, the response rate and demographics of the population who completed it, and comparison of responses of Geraldton residents with those in the NCAS will be discussed.

Learning Outcomes It is feasible to administer a shorter survey based on the NCAS, to measure particular attitudes on which to focus violence prevention education for a regional population.

\section{E.002 CYCLE OF VIOLENCE: CHILDHOOD ABUSE AND RISK OF VIOLENCE REVICTIMISATION IN ADULTHOOD}

${ }^{1}$ Mark A Bellis, ${ }^{2}$ Nadia Butler*, ${ }^{2}$ Zara Quigg. ${ }^{1}$ Public Health Wales, Wrexham, UK; ${ }^{2}$ Liverpool John Moores University, Liverpool, UK

10.1136/injuryprev-2021-safety.50

Interpersonal violence is a serious threat to the attainment of the Sustainable Development Goals (SDGs). A public health approach to violence prevention is crucial, and addressing risk factors is a key priority. Global research has demonstrated that childhood adversity increases risk of a range of poor outcomes across the lifecourse. This study used data from a nationally representative survey of household residents $(n=21,845)$, to examine the impact of childhood abuse (physical, sexual and psychological abuse, and witnessing domestic violence) on risk of adulthood violence revictimisation (physical assault (PA), intimate partner violence (IPV), and sexual violence (SV)).

Compared to individuals who experienced no child abuse, those who experienced one type were, twice as likely to experience PA, and three times as likely to have experienced IPV and/or SV. Individuals who experienced multiple types were three, six and seven times more likely to experience PA, IPV, and SV, respectively. After controlling for the number of types experienced, associated types differed by adult violence outcome; child psychological and physical abuse were associated with IPV; psychological and sexual abuse with SV; and psychological abuse with PA.

Findings from the study will be presented with consideration of strategies to prevent and respond to child abuse and the potential downstream effect on preventing interpersonal violence across the lifecourse and achieving the SDGs. With adulthood victimisation likely to compound the already detrimental effects of childhood abuse, and given that many associated outcomes represent adversities for the next generation, breaking the cycle of violence represents a critical priority.

\section{E.003 BURDEN OF SUICIDE IN NEPAL: AN ANALYSIS OF POLICE RECORDS}

${ }^{1}$ Bhagabati Sedain Silwal ${ }^{*},{ }^{2}$ Puspa Raj Pant,. ${ }^{3}$ Yogendra Bahadur Gurung. ${ }^{1}$ Tribhuvan University Padmakanya Multiple Campus, Kathmandu, Nepal; ${ }^{2}$. Nepal Injury Research Centre (NIRC) 2 University of the West of England (UWE Bristol), Bristol, UK; ${ }^{3}$ Tribhuvan University Central Department of Population Studies, Kathmandu, Nepal

\subsection{6/injuryprev-2021-safety.51}

Background Suicide is a complex human behaviour which might be initiated with an onset of psychic pain. It is the leading cause of unnatural deaths in Nepal, and the number is rising continuously. Due to issues regarding the legality, social stigma, and logistical problems, there is an underreporting of suicide cases in Nepal.

Methods We studied the records of Nepal Police for five years between 2015 and 2019. Access to the dataset was obtained through institutional research collaborations. The socio-demographic variables for each individual case of deaths were extracted and analysed.

Results During the five years, 25,316 cases of suicidal deaths were recorded; an average of 14 people each day. The data revealed that people aged 19 to 35 years accounted for almost $60 \%$ of all suicide deaths. Overall, there is an increase in suicidal deaths by $33 \%$ in 2019 compared to 2015 whereas the increase in females was $200 \%$. Hanging was the most common $(70.13 \%)$ method followed by the consumption of poison $(26.55 \%)$.

Conclusion Suicide is a serious but neglected public health problem in Nepal. It has been found that adolescents, youths, and females are the most vulnerable population for suicide. The findings warrant an urgent need for suicide prevention in Nepal.

Learning Outcomes Police records are currently the only reliable source for information regarding suicides occurring within the nation. There still may be cases that might not have been recorded. Large-Scale researches can identify factors associated with suicides in Nepal in order to inform prevention interventions.

\section{E.004 ZERO SUICIDE HEALTHCARE: PROGRAM THEORY TO GUIDE EVALUATION FOR SAFER SUICIDE CARE}

${ }^{1}$ Sue Murray*, ${ }^{2}$ Alan Woodward*. 'Zero Suicide Institute of Australasia, Balmain, Australia; ${ }^{2}$ Alan Woodward Consulting, Kiama, Australia

10.1136/injuryprev-2021-safety. 52

Context The World Health Organisation estimates one person dies by suicide every 40 seconds. In Australia it's estimated around $20 \%$ of suicides are people who have been in the Australian healthcare system. This is not an acceptable outcome for modern healthcare systems.

Process The Zero Suicide Healthcare (ZSH) framework has been adopted in many developed countries, comprised of seven elements designed to build organisational capability, improve clinical practice and create better service pathways for recovery.

Analysis ZSH draws on techniques of quality management and continuous improvement. It implicitly assumes suicide prevention can be addressed in health care settings in the same way, and with the same absolute improvements, as has been done in wound management, infection control and medication management. It advances evidence-based practice in suicide prevention as a standardised and systematic reform. It encourages the use of data and measurement to monitor performance for continuous improvement within healthcare settings.

However, there is no single source of understanding of how the elements of the ZSH framework interact to achieve outcomes. The development of the Theory of Change for ZSH provides greater clarity about the overall design of the framework using program theory. This will also underpin the evaluation of $\mathrm{ZSH}$ across projects.

Outcomes A model will be presented demonstrating alignment to the $\mathrm{ZSH}$ framework and giving a consistent approach to 
evaluation and monitoring of implementation and supported by standardised data collection.

Learning Outcomes Increased understanding of Theory of Change as it applies to the $\mathrm{ZSH}$ framework.

\section{E.005 TOXICOVIGILANCE FOR SUICIDE PREVENTION FOLLOWING INTERNET PROMOTION OF SODIUM NITRITE}

\author{
1,2,3 Jared Brown*, ${ }^{1}$ Ingrid Berling, 1,3Thanjira Jiranantakan, ${ }^{1}$ Nicholas Buckley, \\ ${ }^{1}$ Andrew Dawson. ${ }^{1}$ NSW Poisons Information Centre, Sydney, Australia; ${ }^{2}$ UNSW, Sydney, \\ Australia; ${ }^{3}$ NSW Ministry of Health, Sydney, Australia
}

10.1136/injuryprev-2021-safety.53

Background There are limited epidemiological studies on emerging trends in suicide methodology relating to Internet promotion of toxic substances. We investigated time trends and demographic characteristics of deliberate self-poisonings with sodium nitrite/nitrate following Internet promotion for euthanasia in 2017 and a cluster of poisonings.

Methods Retrospective observational study of the National Coronial Information System (2000 - June 2020), Poisons Information Centres, toxicology services (2004 - June 2020) and a scoping review including Embase and MEDLINE (2000 - June 2020) for deliberate self-poisonings with sodium nitrite/nitrate. We examined survival, date, gender, age, setting, geographical location, history of a terminal or psychiatric illness, product.

Results We identified 66 deliberate self-poisonings, who were mostly male (65\%) with a wide age distribution (median: 44 years; IQR: 24-66 years; range: 16-92 years; mode: 20-29 years). The majority had a fatal outcome (80\%). A sudden and sustained step-increase in poisonings was seen from September 2017 (and the first death). Most cases (83\%) had a psychiatric illness and no terminal illness (91\%). There were 33 unique cases (mostly young adults) identified in the scoping review from eight countries.

Conclusions The promotion of suicide methodology was associated with a dramatic change in harms from sodium nitrite/ nitrate in the past two decades. The signal generated by poisons centre cases was confirmed using national coronial data and pooled poisoning data.

Learning Outcomes State public health actions to date have focused on means restriction, improved antidote stocking and clinical education. National and international collaboration is needed for monitoring promoted lethal substances.

\section{F - Safe Communities, March 23, 2021}

\section{F.001 SAFE AND BARRIER FREE MODEL CITY DEVELOPMENT IN PINDAYA TOWARDS SDG 2030}

${ }^{1}$ Aye Moe Moe Lwin*. ${ }^{1}$ WHO Country Office, Yangon, Myanmar; ${ }^{2}$ National Rehabilitation Hospital, Yangon, Myanmar; ${ }^{3}$ Township Hospital, Pindaya Township, Myanmar

10.1136/injuryprev-2021-safety.54

Context This project was agreed with community at Pindaya City, place of Danu ethnic tribe from Myanmar, to be long term continuing project from 2019-2030 for achieving Universal Health Coverage for four target populations of persons with disability (PWDs), elderly, children and women and five Sustainable Development Goals.

Process The community agreed during capacity building of key stakeholders that investing for safe and barrier free environment and community-based rehabilitation (CBR) support was more economical and sustainable. Barrier free hospital and health clinics followed by improving services and infrastructure of the public places to be barrier free.

Analysis The mobilization of financial resources from Danu self-administrative department and Township Development Committee were main mechanisms for financial resources. Technical support on capacity building for safety and accessibility audit for vulnerable people was provided by WHO and Myanmar Independent Living Initiative. Setting up of comprehensive center for violence survivors, CBR program for PWDs and establishment of management committee to plan and implement were important activities.

Outcomes The community can build their own future they want for safety, accessibility, participation and economy. Nearly 80 to $90 \%$ of targets achieved after first year. As the community develops, benefit will also be for all people, not only vulnerable groups.

Learning Outcomes Sustainability is promising when local government and community pursue with own vision and goals, planning own financial resources and capacity building for safety and barrier free.

\section{F.002 EXPLORING SAFE AND SUSTAINABLE CITIES}

Kelly Keena*. Underwriters Laboratories, Northbrook, USA

\subsection{6/injuryprev-2021-safety.55}

UL Xplorlabs is an online virtual safety science resource for secondary students and their teachers. Based on the safety research being conducted at Underwriters Laboratories, UL Xplorlabs investigates the science behind the safety in online and hands-on investigations.

In 2019, UL Xplorlabs launched a new module under the theme Safe and Sustainable Cities. The first in the series is Extraction to Ewaste: The Lithium-ion Battery Supply Chain. In this module, participants investigate a mobile phone's power source: each step in a Li-ion pouch cell's production from raw minerals to when the phone arrives in our hands. Then, the module explores the global issue of e-waste as the phones break or become obsolete.

As a part of the UN Sustainable Development Goal (SDG) \#11 on Sustainable Cities and Communities, Xplorlabs supports youth understanding and solutions for e-waste, a global safety issue.

\section{F.003 30-YEAR ANALYSIS OF DESIGNATED INTERNATIONAL SAFE COMMUNITIES}

Dale Hanson*. James Cook University, Glenelg South, Australia

\subsection{6/injuryprev-2021-safety.56}

Background The International Safe Communities movement was established in 1986. It developed into an international collaborative network comprised of the WHO CCCSP/ISCCC, regional and national safe community organisations, support centres and communities. 